\title{
Variability in Mass Spectrometry-based Quantification of Clinically Relevant Drug Transporters and Drug Metabolizing Enzymes
}

Christine Wegler, ${ }^{\dagger}{ }^{\ddagger}$ Fabienne Z. Gaugaz, ${ }^{\dagger}$ Tommy B. Andersson, ${ }^{\ddagger}$ Jacek R. Wiśniewski, ${ }^{\S}$ Diana Busch, Christian Gröer, "Stefan Oswald," Agneta Norén, ${ }^{\perp}$ Frederik Weiss," Helen S. Hammer," Thomas O. Joos," Oliver Poetz, ${ }^{\#}$ Brahim Achour, ${ }^{\nabla_{\odot}}$ Amin Rostami-Hodjegan, ${ }^{\nabla}$ Evita van de Steeg, Heleen M. Wortelboer, and Per Artursson* ${ }^{* \dagger}$

${ }^{\dagger}$ Department of Pharmacy, Uppsala University, Uppsala 75123, Sweden

${ }^{\ddagger}$ Cardiovascular and Metabolic Diseases, Innovative Medicines and Early Development Biotech Unit, AstraZeneca R\&D, Mölndal 431 50, Sweden

${ }^{\S}$ Biochemical Proteomics Group, Department of Proteomics and Signal Transduction, Max Planck Institute of Biochemistry, Martinsried 82152, Germany

"Center of Drug Absorption and Transport, Department of Clinical Pharmacology, University Medicine of Greifswald, Greifswald 17489, Germany

${ }^{\perp}$ Department of Surgical Sciences, Uppsala University, Uppsala 75185, Sweden

${ }^{\#}$ NMI Natural and Medical Sciences Institute, University of Tübingen, Reutlingen 72770, Germany

${ }^{\nabla}$ Centre for Applied Pharmacokinetic Research, University of Manchester, Manchester M13 9PL, United Kingdom

- TNO (Netherlands Organization for Applied Scientific Research), 3700 AJ Zeist, Netherlands

\section{Supporting Information}

ABSTRACT: Many different methods are used for mass-spectrometry-based protein quantification in pharmacokinetics and systems pharmacology. It has not been established to what extent the results from these various methods are comparable. Here, we compared six different mass spectrometry-based proteomics methods by measuring the expression of clinically relevant drug transporters and metabolizing enzymes in human liver. Mean protein concentrations were in general quantified to similar levels by methods using whole tissue lysates. Methods using subcellular membrane fractionation gave incomplete enrichment of the proteins. When the enriched proteins were adjusted to levels in whole tissue lysates, they were on average 4fold lower than those quantified directly in whole tissue lysates. The differences in protein levels were propagated into differences in predictions of hepatic clearance. In conclusion, caution is needed when comparing and applying quantitative proteomics data obtained with different methods, especially since membrane fractionation is common practice for protein quantification used in drug clearance predictions.

KEYWORDS: drug transporters, drug metabolizing enzymes, membrane proteins, protein quantification, targeted proteomics, label-free proteomics

\section{INTRODUCTION}

Cellular proteins define phenotype and function by responding dynamically to environmental and genetic factors. Describing and understanding the quantitative proteome is a central and fundamental challenge in biology. ${ }^{1-4}$ Currently, most quantitative proteomics strategies are based on bottom-up mass spectrometry approaches, where proteins are digested to peptides prior to analysis. ${ }^{5}$ However, these methods differ from one laboratory to another. Many of these proteomic methods are not yet evaluated and standardized and the comparability of the generated data are challenged by differences in sample preparation, digestion procedure, and
LC-MS/MS analysis. Samples are prepared from whole tissue lysates $^{6-9}$ or from isolated subcellular fractions. ${ }^{10-16}$ The mass spectrometry instruments can be triple quadrupoles, quadrupole time-of-flight, and quadrupole orbitraps. ${ }^{17}$ The targeted approaches use isotope labeled standards ${ }^{18,19}$ while global approaches can use label-free quantification. ${ }^{20,21}$ It has not yet been established to what extent the variability in reported

Received: May 3, 2017

Revised: July 28, 2017

Accepted: August 2, 2017

Published: August 2, 2017 


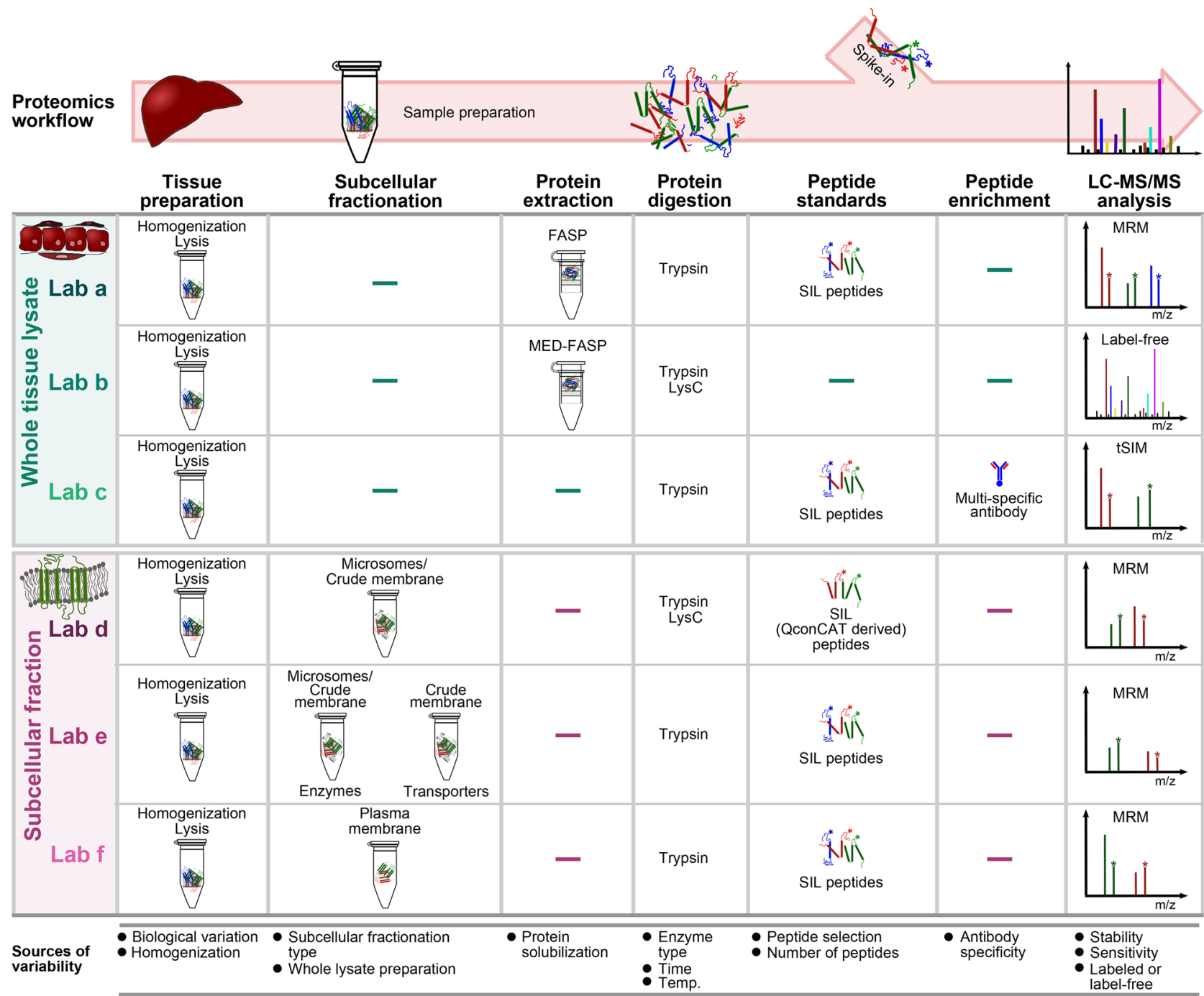

Figure 1. Illustration of methods included in the study. Identical human liver tissue samples from ten individuals were distributed to six laboratories. In house-methods were employed for tissue preparation and protein quantification. Results were finally compiled and analyzed in one of the laboratories. MED-FASP: multi enzyme digestion-filter aided sample preparation; SIL peptides: stable isotope labeled peptides; MRM: multiple reaction monitoring; tSIM: targeted single ion monitoring; QconCAT: quantification conCATemer.

protein quantities is related to the choice of proteomic strategy. $^{22-25}$ Previous meta-analyses of studies quantifying proteins essential for drug disposition, such as hepatic drug transporting and metabolizing proteins, revealed a larger variability than could be accounted for by biological variation. This suggests that methodological differences also contributed to the variability. ${ }^{26-29}$

Here we investigated how the protein quantification varied between different methods. Six laboratories that had developed their own in house-methods for quantification of drug transporting proteins and drug metabolizing enzymes were included in the study. These liver proteins are of particular importance in drug discovery and development since they participate in disposition, metabolic processes, and elimination of xenobiotics, and hence determine drug exposure. They are also used for parametrization of bottom-up cellular or systems pharmacology models. We therefore also investigated the impact of the observed variability in protein quantities on clearance prediction of atorvastatin. Our study indicates that protein levels obtained from different proteomics methods can vary considerably, especially when using membrane fractiona- tion. This study therefore demonstrates that caution is needed when comparing and applying quantitative proteomics data, and that evaluation and standardization of proteomics methods are warranted.

\section{EXPERIMENTAL SECTION}

Human Liver Tissue. Normal human liver tissues were obtained from ten liver resections carried out at the Department of Surgery, Uppsala University Hospital (Uppsala, Sweden). The study was approved by the Uppsala Ethical Review Board (ethical approval no. 2009/028 and 2011/037). Donors gave their informed consent. Donor demographics are summarized in Table S1. Liver tissue samples were snap-frozen (in methylbutane on dry ice and ethanol) immediately after resection and stored at $-150{ }^{\circ} \mathrm{C}$. The snap-frozen samples were divided under liquid nitrogen and distributed frozen on dry ice to the six participating laboratories. In each laboratory, tissue samples were prepared and analyzed in accordance to in houseproteomics methodologies.

Proteins for Quantification. Liver proteins important for drug transport and metabolism were included in this 
quantitative analysis. The proteins were selected for the widest possible coverage of proteins essential for drug disposition, with respect to available in house-methods across the six laboratories. In total, five hepatic uptake (SLC22A1, SLCO1A2, SLCO1B1, SLCO1B3, and SLCO2B1) and five efflux (ABCB1, ABCC2, ABCC3, ABCG2, SLC47A1) transmembrane transporters were included in the study. In addition, nine phase I- (CYP1A2, CYP2B6, CYP2C8, CYP2C9, CYP2C19, CYP2D6, CYP3A4, and CYP3A5) and four phase II- (UGT1A1, UGT1A3, UGT2B7, UGT2B15) drug metabolizing enzymes localized in the endoplasmic reticulum were quantified in four laboratories (Figure 3a). The quantified proteins and proteotypic peptides used for the quantification are listed in Table S2 and Table S3, respectively.

Proteomic Methods. Six different liquid chromatographymass spectrometry-based proteomics methods were applied to quantify membrane proteins in ten liver samples. Briefly, proteins were solubilized from whole tissue lysates in three of the laboratories (Laboratories a, b, and c), before digestion (Figure 1 and Table S4 give a schematic overview of the methodologies). In the other three laboratories (Laboratories $\mathrm{d}$, e, and $\mathrm{f}$ ), total/crude membrane or plasma membrane fractions were isolated as an enrichment step prior to protein digestion, in order to concentrate the proteins of interest. Lab $\mathrm{c}$ isolated the peptides of interest using immunoprecipitation from the whole cell digests prior to LC-MS analysis, giving less complex digests. Five laboratories quantified the proteins using targeted proteomics, with stable isotope labeled standards (Lab a, c, e, and $\mathrm{f}$ ) or concatenated synthetic peptides, QconCAT (Lab d). One laboratory (Lab b) used a shotgun, label-free approach (total protein approach, TPA) to quantify the proteins. A more detailed description of the proteomic methods used by each laboratory can be found in Supporting Information Methods, together with the amount of used tissue for each method (Figure S1).

Comparative Data Analysis. Intralaboratory Reproducibility. Tissue preparation and analysis were performed at two independent occasions with duplicate tissue samples from the same ten individuals. The protein concentrations obtained from each run were compared to measure the within-laboratory reproducibility by calculating the average fold error (AFE) and absolute average fold error (AAFE; see Supporing Information Methods). ${ }^{30}$ The AFE was used to assess if the protein levels were measured higher (or lower) in one of the runs, while AAFE was used to assess the variability of the duplicate measurements (the spread of the mean fold values from the reference value at 1 , i.e., identical results). ${ }^{30}$

Comparison between Laboratories. Correlations within and across laboratories were determined by Pearson's productmoment correlation coefficients $(r)$ calculated on log-values of protein concentrations. The consistency of the order of protein levels across the methods was investigated where the log-values of protein concentrations, for each protein, were compared across the laboratories. Good correlation was considered for comparisons with correlation coefficients above $0.79(p<0.05$ after Bonferroni correction for multiple comparisons). The influence of reference peptides on protein quantification was investigated by comparing proteins quantified with the same reference peptide (matched) or proteins quantified with different reference peptides (unmatched), across the laboratories. In the label-free approach, proteins are quantified based on several peptides and were included in the comparison if one of the numerous peptides matched the peptide used in the targeted approach.

Scaling Protein Concentrations from Enriched Subcellular Fractions to Whole Tissue. Different subcellular fractionation procedures (such as membrane and microsomal enrichment) were conducted in Laboratories $\mathrm{d}$, $\mathrm{e}$, and $\mathrm{f}$ to enable quantification of the proteins. These protein levels were quantified in $\mathrm{fmol} / \mu \mathrm{g}$ membrane protein. To enable direct comparisons with Laboratories $\mathrm{a}, \mathrm{b}$, and $\mathrm{c}$ where proteins were quantified in $\mathrm{fmol} / \mu \mathrm{g}$ total tissue protein, the protein concentrations in the subcellular fractions required scaling to the corresponding whole tissue. By assuming full recovery in the fractionation processes, the results were scaled by applying eq 1 ,

$$
\begin{aligned}
& \text { Protein conc }_{\text {whole tissue }}= \text { Protein conc } \text { membrane fraction } \\
& \times \frac{\text { Total protein amount }}{\text { membrane fraction }} \\
& \text { Total protein per tissue } \times \text { Tissue weight }
\end{aligned}
$$

where Protein conc $_{\text {whole tissue }}$ is the scaled concentration of the protein of interest in the whole tissue $(\mathrm{fmol} / \mu \mathrm{g}$ total tissue protein), Protein conc $_{\text {membrane fraction }}$ is the concentration of the protein of interest measured in the isolated fraction $(\mathrm{fmol} / \mu \mathrm{g}$

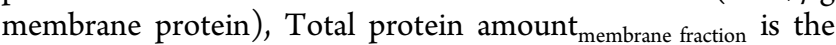
total amount of protein measured in the cell fraction $(\mu \mathrm{g})$, while Tissue weight is the amount of tissue $(\mathrm{mg})$ used for the enriched cell fraction preparation. Finally, Total protein per tissue is the amount of homogenate protein per mg of liver ( 88 $\pm 14 \mu \mathrm{g} / \mathrm{mg} ; n=12^{31}$ ).

Predicting Uptake Clearance, $C L_{\text {int,uptaker }}$ of Atorvastatin. The contribution of three transporters, SLCO1B1, SLCO1B3, and $\mathrm{SLCO} 2 \mathrm{~B} 1$, to the relative $\mathrm{CL}_{\text {int,uptake }}$ of atorvastatin was estimated using a static, mathematical model described previously. ${ }^{31}$ In vitro kinetic and in vitro proteomics data were obtained from Karlgren et al. Thus, the only variable in the model was the protein levels quantified in the liver tissues, which enabled the evaluation of the effect of different protein levels obtained from the six methods. This allowed calculation of $\mathrm{CL}_{\text {int,uptake }}$ expressed in relative units, since different methods were used to quantify the proteins in vitro ${ }^{31}$ and in the liver samples in this study. All calculations were made in Microsoft Excel (version 14.0.7128.5000), while box plots, figures, and statistical tests were made with GraphPad Prism (version 7.0).

\section{RESULTS}

Variability in reported protein concentrations can arise from multiple sources. These include procedures for tissue resection, sample preparation, analysis methodology, as well as true biological variation between individuals. Here, liver tissue samples from ten donors were snap-frozen in a single laboratory under standardized conditions, and identical samples were distributed to the six participating laboratories. Thus, the observed variability should arise primarily from the different procedures and equipment used for sample preparation and proteomic analysis (see in house-methods in Figure 1), rather than from tissue procurement and biological variation.

Intralaboratory Reproducibility. To assess the reproducibility of the results within the laboratories each participating laboratory prepared and analyzed samples from ten donors at two different occasions. The reproducibility was compared by determining the average fold error (AFE) and absolute average fold error (AAFE) within the laboratories. The AFE was used to assess if the protein levels were measured higher (or lower) 

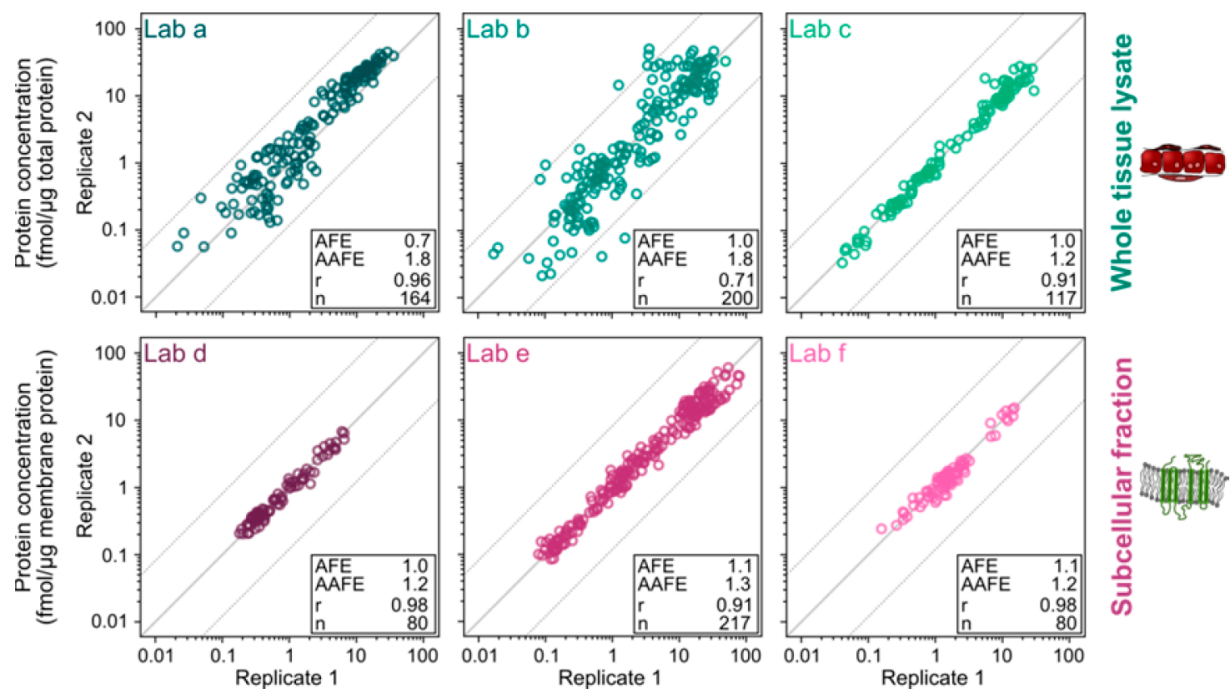

Figure 2. Intralaboratory reproducibility. Sample preparation and protein quantification in the ten liver samples were performed at two independent occasions. The reproducibility of the methods was determined by calculating AFE and AAFE for the duplicate runs. AFE: average fold error; AAFE: absolute average fold error; $\mathrm{r}$ : coefficient of variation; $\mathrm{N}$ : number of proteins in the ten liver samples measured in each of the independent runs.

in one of the runs, while AAFE was used to assess the variability of the duplicate measurements. ${ }^{30}$ High reproducibility (AFE and AAFE close to 1) was obtained when sample complexity was reduced after concentration of membrane proteins by subcellular fractionation (Laboratories $\mathrm{d}$, e, and $\mathrm{f}$ ) (Figure 2), as well as after antibody-based peptide enrichment in whole tissue digests (Lab c). Slightly different results were obtained in the remaining two laboratories that quantified peptides from whole tissue digests (Laboratories a and b). The AFE within Lab b, which used a label-free, total protein approach (TPA), was at terms with the other laboratories (Laboratories $c, d, e$, and $\mathrm{f}$ ). However, proteins in Lab a were on average 1.5-fold higher in one of the runs. The differences of the duplicate measurements (AAFE) were larger in Laboratories a and b, compared to the laboratories that used enrichment procedures. This was most likely related to higher complexity (larger number of proteins) in the whole tissue digest samples.

Transporter Protein Levels in Isolated Subcellular Fractions. Human uptake and efflux transporters (four ABC and six SLC-transporters) were quantified in the laboratories (Figure 3a, Table S2). Three of the laboratories quantified the transporters in isolated membrane fractions after subcellular fractionation processes, yielding crude (or total) membrane (Laboratories e and d) or plasma membrane fractions (Lab f). These subcellular protein concentrations were expressed in $\mathrm{fmol} / \mu \mathrm{g}$ membrane protein. On average, proteins were quantified to similar levels in the different subcellular fractions (Laboratories d, e, and f; Figure $3 \mathrm{~b}$ and $\mathrm{c}$ ), and were in general comparable to previously reported values from subcellular fractions (Figure S2a). However, low degrees of enrichment were observed, with for example only up to 3 -fold enriched levels in crude membrane fractions (Lab d and e), compared to in the whole tissue digests (Laboratories a, b, and c; Supporting Excel File 1, Figure S2b). Various estimates of the membrane proteome indicate that the membrane fraction contributes between 16 and $25 \%$ of the total cellular protein. ${ }^{32-35}$ Thus, with the assumption of full recovery, fractionation procedures for crude (or total) membrane proteins are expected to yield 4-6 fold enrichment. Surprisingly, for isolated plasma membrane fractions (Lab f) only a 6-fold enrichment was obtained (Figure S2b), indicating a substantial loss of plasma membrane proteins (or contamination of proteins from other subcellular fractions) during the subcellular fractionation. Plasma membrane proteins are assumed to make up $1-3 \%$ of the total cellular protein, ${ }^{22,35,36}$ and isolated plasma membrane fractions are therefore expected to be concentrated 30-100fold.

Comparison of Mean Protein Levels across All Laboratories. The lower recovery from the membrane purification processes leads to an underestimation of the protein quantities. This was evident when the enriched protein levels in the isolated subcellular fractions were corrected to those in whole tissue, with the assumption of full recovery of membrane proteins in the fractionation procedures, using eq 1. The correction enabled direct comparison of the quantified membrane proteins (transporters and enzymes) across all laboratories, and the corrected protein levels should, in theory based on above assumptions, reach the same levels as those obtained directly from whole tissue digests.

Comparison of Transporter Protein Levels. The corrected transporter levels obtained from Lab f, using plasma membrane fractionation, were consistently 10 -fold lower compared to levels from the other methods $(p<0.05$ in Mann-Whitney test, Figure $4 \mathrm{~b}$ ). For example, Lab $\mathrm{f}$ reported up to $120-, 80-$, and 50-fold lower levels of SLCO1B1, SLCO1B3, and ABCC3, respectively, compared to the other laboratories $(p<0.05$ in Kruskal-Wallis test followed by Dunn's multiple comparisons test, Tables S5 and S6). The differences in mean concentrations across the other five laboratories were lower: 7-, 6-, and 3-fold, for the three proteins. The 120-fold lower levels of SLCO1B1 (Lab f) could be explained by the low recovery from the membrane purification process, but also partly by the peptide used for the protein quantification. The peptide sequence used in Lab $\mathrm{f}$ covered the transmembrane domain in position 402409 of the protein, which should be avoided since it can lead to poor peptide recovery ${ }^{24}$ resulting in lower quantified levels of the protein. While analysis of whole tissue lysates gave more consistent quantification, the complexity of the samples reduced the sensitivity. Thus, some low abundance transport proteins were only detected after subcellular fractionation. For 


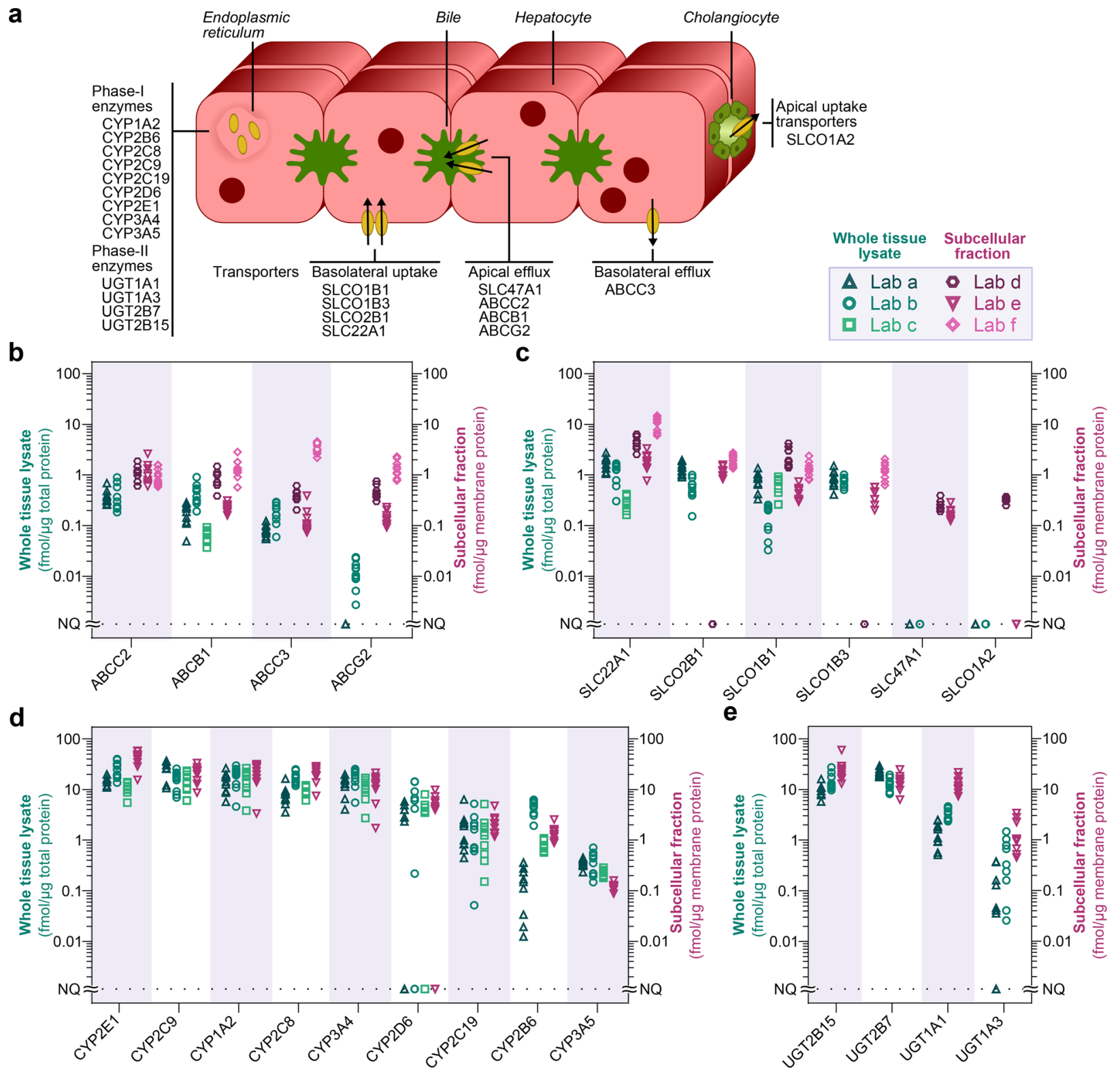

Figure 3. Comparison of protein levels across laboratories. Subcellular localization of proteins analyzed in the study (a). Protein levels (fmol $/ \mu \mathrm{g}$ total protein from whole tissue lysates and $\mathrm{fmol} / \mu \mathrm{g}$ membrane protein from subcellular fractions) of ABC-transporters (b), SLC-transporters (c), CYPenzymes (d), and UGT-enzymes (e) for the ten liver samples were compared between each laboratory. NQ: not quantified, proteins could not be quantified with the applied methods..

instance, the low abundant proteins SLC47A1 and SLCO1A2 (mean values of 0.06 and $0.05 \mathrm{fmol} / \mu \mathrm{g}$ total protein, respectively) could only be quantified by Laboratories $\mathrm{d}$ and e (only SLC47A1) after enrichment procedures. In contrast, ABCG2 (mean value of $0.03 \mathrm{fmol} / \mu \mathrm{g}$ total protein) could also be detected in whole tissue digests by Lab b.

Comparison of Enzyme Protein Levels. Essential drug metabolizing enzymes (CYPs (Cytochrome P450s) and UGTs (UDP-glucuronosyltransferases)) were also quantified in four of the six laboratories (Laboratories a, b, c, and e). The enzyme levels determined in whole tissue digests (Laboratories $a, b$, and c) were comparable, with only a 2 -fold average difference. Similar to the transporter proteins, the enzymes were poorly enriched in subcellular fractions in Lab e. Correcting the levels to whole tissue resulted in on average 6-fold lower levels compared to in whole tissue digests. However, the overall fold variation across the four laboratories was smaller for the higher abundant enzymes, compared to what was observed for the transporters (Figure 4c, Table S5). As for the transporters, not all proteins could be quantified by all laboratories. For instance, Lab a could not quantify the protein levels of UGT1A3 in all donor samples, since they were close to the limit of quantification. CYP2D6 could only be quantified in seven out of ten liver samples in all four laboratories, indicating very low protein levels in these samples. This could however be due to genetic variation, with CYP2D6 being a polymorphic protein with null-alleles in $24 \%$ of the population. ${ }^{37}$

Correlation of Protein Expression in the Ten Liver Donors. Despite that some of the laboratories showed large differences in the mean levels of some proteins, we asked ourselves if the different methods provided consistent results in the order of the ten liver samples for each of the 23 investigated 
a

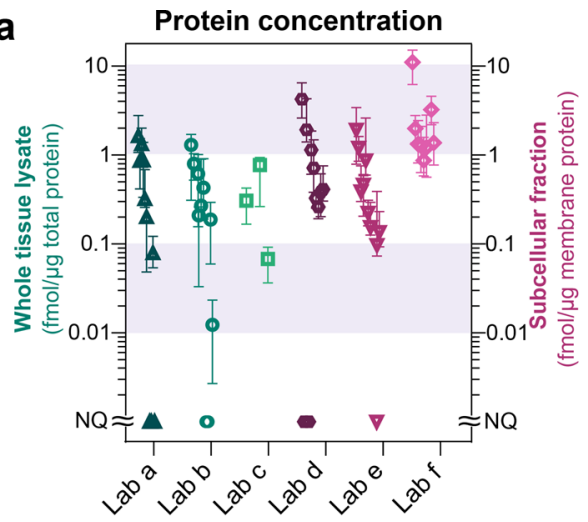

b

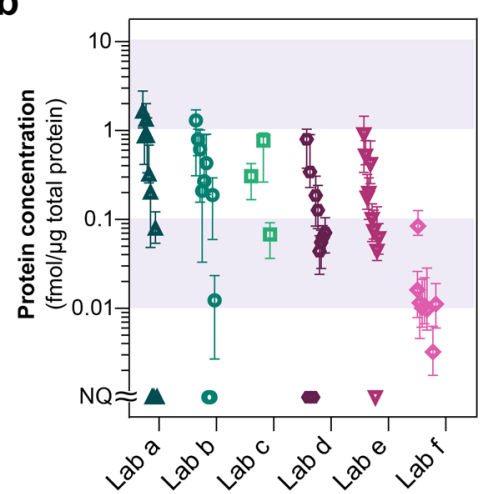

C

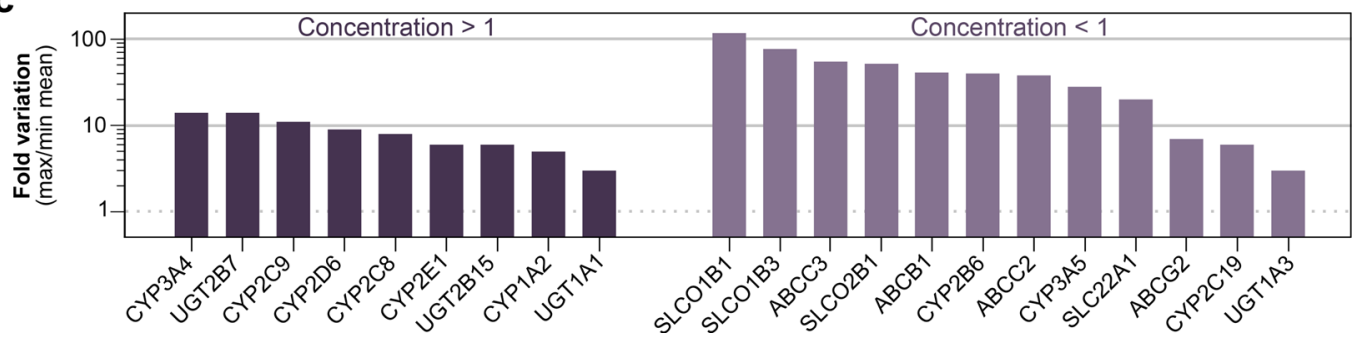

Figure 4. Comparison of transporter protein levels in whole tissue digests $(\mathrm{fmol} / \mu \mathrm{g}$ total protein; Lab a-c), in concentrated subcellular fractions $(\mathrm{fmol} / \mu \mathrm{g}$ membrane protein; Lab d-f) (a), and levels after correction to those in whole tissue lysate (fmol/ $\mu \mathrm{g}$ total protein) using eq 1 (b). After adjusting to whole tissue, large fold differences between the different methods were demonstrated (c). NQ: not quantified, proteins could not be quantified with the applied methods.

proteins (i.e., if a sample with the highest expression of a certain protein in one laboratory also reported the highest expression across all laboratories). The distribution of the Pearson's correlation coefficients for all proteins for all laboratory comparisons is shown in Figure 5a and Figure S3. Only onethird of the correlations were significantly correlated across the laboratories $(r>0.79 ; p<0.05$ after Bonferroni correction for multiple comparisons, Figure S3). For most of the proteins the order of the ten liver samples varied between paired laboratories. For instance, one individual was found to have the highest expression of $\mathrm{ABCB} 1$ in Lab e, but the lowest in Lab $b$ (resulting in $r=-0.48$, Figure $5 b$ ). However, the same protein showed a good correlation between Laboratories $b$ and c $(r=0.94)$ (Figure 5b). Similarly, the correlation coefficients for SLC22A1 ranged from poor correlation between Laboratories a and $\mathrm{f}(r=0.46)$ to excellent correlation between Laboratories $\mathrm{c}$ and e $(r=0.94$. Figure 5c).

We also investigated potential reasons for the poor correlations. First, we analyzed if these correlations were influenced by the protein abundances (Figure 5d). To our surprise, we found that this was not the major reason for the poor correlations as high $r$-values (above 0.79 ) were observed across a wide range of protein concentrations ( 0.17 to 20.5 $\mathrm{fmol} / \mu \mathrm{g}$ total protein, Figure $5 \mathrm{~d}$ ). Second, comparable mean protein levels between two laboratories did not result in better correlations. High $r$-values (above 0.79) were obtained irrespective of fold differences in protein concentrations (up to 40-fold, Figure 5e). We also analyzed if differences in proteotypic peptides used for quantification had an impact on the correlations (Supporting Information Results). However, differences in the used peptides could not explain the wide range in correlations: the $r$-values ranged from -0.60 to 0.98 , and -0.70 to 0.98 , for proteins quantified with matched peptides and unmatched peptides, respectively (Figure 5f, Supporting Information Results and Figure S4). Overall, these results show that the protein concentrations were in general poorly correlated in the ten liver samples across the different laboratories.

Impact of Protein Quantification on Pharmacokinetic Predictions. Finally, we assessed the impact of variable protein quantification on predictions of a commonly used pharamacokinetic parameter, the intrinsic liver clearance $\left(\mathrm{CL}_{\text {int,uptake }}\right)$. Protein levels obtained from the different methods were used in a previously publised static mathematical model for estimating the maximal uptake clearance, $\mathrm{CL}_{\text {int,uptake, of atorvastatin. }}{ }^{31}$ All parameters in vitro were used as previously reported and kept constant in the comparisons, so that only the effect of variable liver protein quantities obtained in this study could be illustrated. Since different methods were used for quantifying proteins in the in vitro-system and in the livers, the $\mathrm{CL}_{\text {int,uptake }}$ were expressed in relative units. Atorvastatin is actively transported by SLCO1B1, SLCO1B3, and SLCO2B1. ${ }^{31}$ Importantly, since Laboratories $\mathrm{c}$ and $\mathrm{d}$ only quantified SLCO1B3 and SLCO1B1, respectively, these were not included in the final analysis of the overall fold-difference in the predicted relative uptake clearance (cumulative $\mathrm{CL}_{\text {int,uptake }}$ of each SLCO-transporter). The overall predicted uptake clearance in vivo varied on average 2-fold (range 1.3-4 fold; Figure $6 \mathrm{~b}$ ) across the laboratories, with a larger variation when enriched protein levels were corrected to those in whole tissue (7-fold average difference; range 1.8-90 fold; Figure 6c, Figure S5). Together, this demonstrates that interlaboratory differences in protein quantities had a strong influence on the predicted liver clearance.

\section{DISCUSSION}

In predictive pharmacokinetics, drug transporting and metabolizing enzymes are often quantified in subcellular fractions and reported as fmol $/ \mu \mathrm{g}$ membrane protein. The protein quantities 
a

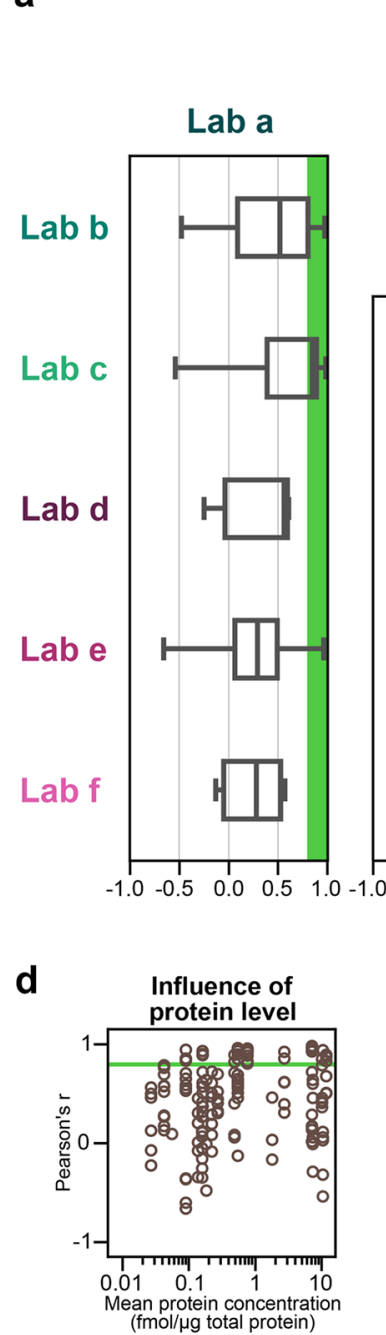

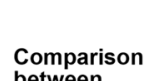
between two labs

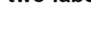

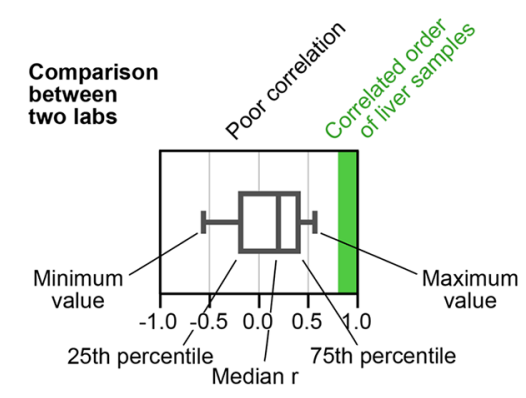

Lab b

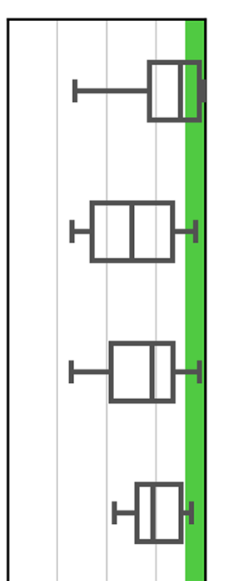

Lab c

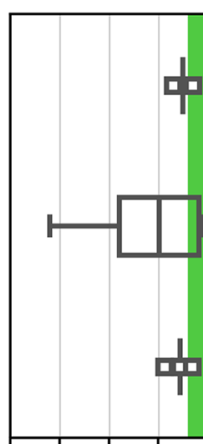

Lab d b
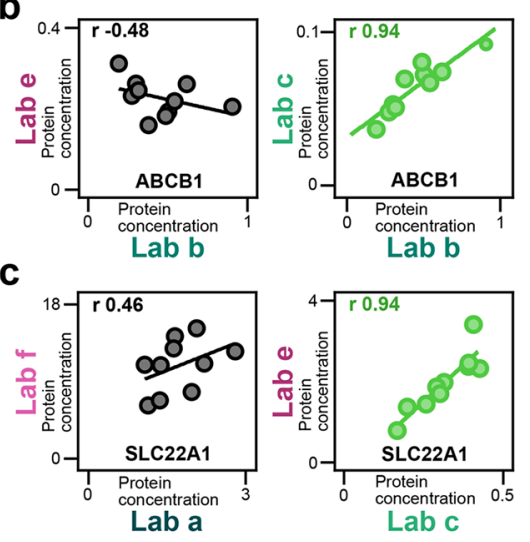

$\begin{array}{lllllllllllllllll}0.5 & 1.0 & -1.0 & -0.5 & 0.0 & 0.5 & 1.0 & -1.0 & -0.5 & 0.0 & 0.5 & 1.0 & -1.0 & -0.5 & 0.0 & 0.5 & 1.0\end{array}$

ofrelations of liver samples
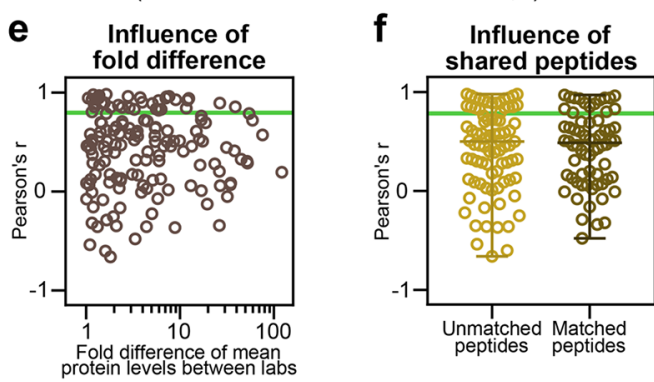

Correlated order of liver samples

Lab e

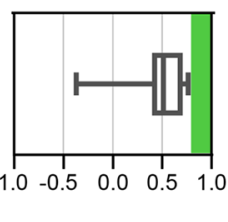

Figure 5. Distribution of correlations of liver samples based on protein expressions across laboratories. The consistency in order of the ten liver samples, for each protein, was compared across the laboratories using Pearson's product-moment correlation. The distribution of correlation coefficients across the laboratories is summarized in boxplots (a). Good correlation (green) was considered for Pearson's product-moment correlation coefficients above 0.79 ( $p<0.05$ after Bonferroni-correction for multiple comparisons). Examples of poor and good correlations of order of liver samples for ABCB1 (b) and SLC22A1 (c) between laboratories are also given. Pearson's $r$-values were not influenced by the quantified abundancy of the protein $(\mathrm{d})$, the magnitude of the fold difference of mean protein levels across the laboratories (e), or whether matched peptides were used for protein quantification (f). $r$ : Pearson's correlation coefficient.

are then used in modeling of in vitro-in vivo correlations. In this study, we compared protein levels of transporters and enzymes obtained in subcellular fractions, with those in whole tissue digests. We found that enrichment of membrane proteins was lower than expected after subcellular fractionation, in particular in the plasma membrane fractions, when compared to protein levels measured in whole tissue digests. This is in agreement with previously demonstrated difficulties in isolating integral membrane proteins in a single membrane fraction, demonstrating lower than expected yields of membrane proteins in the isolated fraction. ${ }^{15,22,34,38}$ This is not only caused by suboptimal membrane fractionation methods, but also by cellular membrane dynamics, ${ }^{39}$ making it difficult to obtain some membrane compartments (e.g., the plasma membrane) in pure form. Very promising, but demanding procedures to obtain maximal sorting of membrane compartments by subcellular fractionation have recently been proposed. ${ }^{35,40}$ Unfortunately, this procedure is cell specific and has so far only been applied to single homogeneous cell lines. It remains to be shown if it is applicable to heterogeneous tissues.

A common way to account for the enrichment of membrane proteins is to use membrane markers, such as ATP1A1 $\left(\mathrm{Na}^{+} /\right.$ $\mathrm{K}^{+}$-ATPase). This protein is predominantly located in the plasma membrane, more specifically in the basolateral membrane of hepatocytes. ${ }^{15,41}$ Unfortunately, our results indicate that each transporter and enzyme is enriched to a different degree, limiting the usefulness of single membrane markers (Table S5 and Supporting Excel File 1). This is in agreement with our recent observation that apical and basolateral plasma membrane proteins are enriched differently during subcellular fractionation of human liver tissue. ${ }^{34}$ An alternative approach to correct for the loss of proteins is to determine protein specific recovery factors. ${ }^{22}$ Unfortunately this is a demanding procedure not applicable for routine use. We conclude that general correction factors are at the present time not sufficiently refined for accurate quantification of individual membrane proteins. 
a

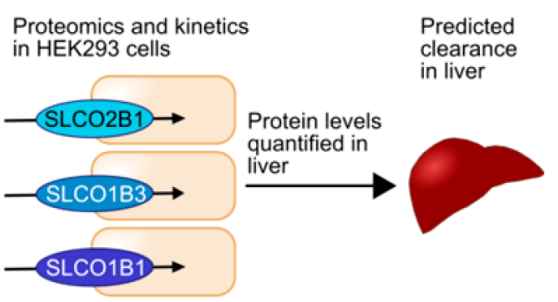

b
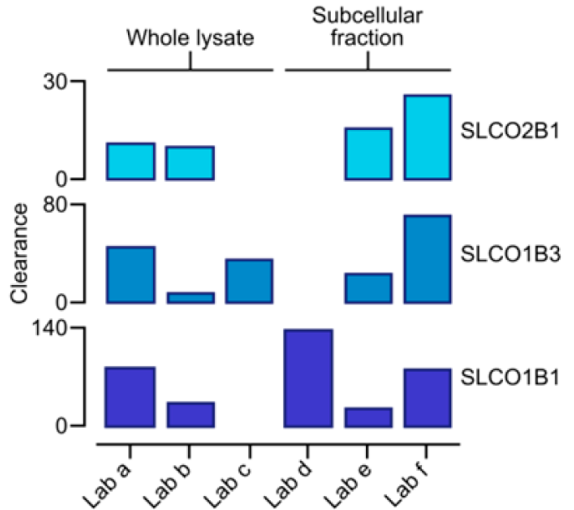

C

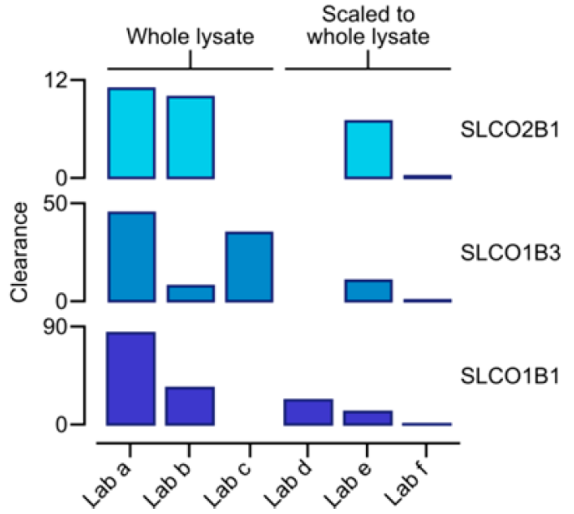

Figure 6. Prediction of hepatic uptake clearance $\left(\mathrm{CL}_{\text {uptake }}\right)$ of atorvastatin. A static mathematical model $^{31}$ was used in predicting the influence of varying levels of SLCO1B1, SLCO1B3, and SLCO2B1, from the laboratories, on atorvastatin clearance (a). The predicted $\mathrm{CL}_{\text {uptake }}$ of atorvastatin by each of the three transporters is based on the protein quantities obtained in Lab a-f $(\mathrm{fmol} / \mu \mathrm{g}$ total protein and $\mathrm{fmol} / \mu \mathrm{g}$ membrane protein) (b) and protein levels corrected to whole tissue (fmol $/ \mu \mathrm{g}$ total protein) (c). The contribution of each transporter is represented as geometrical mean values of calculated clearance from the ten liver samples. Lab $\mathrm{c}$ and Lab d only quantified SLCO1B3 and SLCO1B1, respectively.

Label-free proteomics methodologies have historically been considered less sensitive and reproducible compared to targeted approaches using labeled peptide standards. ${ }^{19}$ Our results show that recent developments (e.g., MED-FASP and TPA) for labelfree proteomics, ${ }^{21}$ together with enhanced instrument sensitivity have improved this method to a level approaching that of targeted proteomics, that uses labeled standard peptides, c.f. Figure 2 (Lab a and b). This observation is supported by a study by IJsselstijn et al., which demonstrated good quantification consistency between a targeted and label-free approach. ${ }^{42}$ One of the benefits of label-free, global approaches is that the protein quantification is based on several peptides, ${ }^{21}$ thus reducing the inherent bias of only using single or a few peptides. Here, an average of $12(3-35)$ unique and razor peptides per protein were used by Lab $b$ for the label-free quantification. The challenge of using single peptide standards was illustrated by the considerably lower quantified levels of SLCO1B1 in Lab $f$, where the chosen peptide spans the transmembrane domain of the protein.

The higher complexity of the whole tissue digests (Laboratories $\mathrm{a}$ and $\mathrm{b}$ ) provides a reasonable explanation for the lower reproducibility compared to when membrane proteins were enriched prior to analysis. Complex biological matrices affect mass spectrometry measurements by several factors, including competition for ionization and signal suppression or enhancement effects. ${ }^{43}$ This was confirmed by Lab c, which demonstrated high reproducibility after reduction of the whole tissue digest complexity by immunoprecipitation of selected peptides with validated antibodies. Although demanding in terms of identification and validation of suitable antibodies for the proteins, once established this approach seems to be an attractive alternative to subcellular fractionation.

The results of this study also have implications for drug development. Following recommendations of FDA and EMA, physiologically based system models are increasingly used to predict clinical pharmacokinetic and dynamics prior to clinical studies of new drug candidates. ${ }^{44,45}$ The pertinence of these models is dependent on the quality of the physiological parameters entered into the models. Among these is protein concentration of the liver transporters and enzymes investigated in this study. Apart from normal biological factors, including genetic variability in the clinical population, an interlaboratory variability of up to 120 -fold was observed for the drug transporting and drug metabolizing proteins in the human liver, when considering protein levels in the whole tissue. This was propagated into large differences in adjusted uptake clearance of atorvastatin across the laboratories. Further, significant effects on the predicted clinical pharmacokinetics and dynamics outcomes are expected. Clearly, this variability would be reduced by more systematic evaluation and standardization of methods for quantitative proteomics. ${ }^{1,16,46}$

The scope of this study was to investigate the variability in protein quantification between proteomics methods currently used in ADME research, and not to identify a "gold standard" method for quantifying membrane proteins. By exploring currently used in house-methods, the variability in protein quantification could be revealed. In addition to the sources of variability compared in this study, other sources of variability such as tissue homogenization, , $3,47-49^{\text {degree of protein }}$ solubilization, ${ }^{49}$ and digestion conditions of proteins (e.g., type of digestion enzyme, digestion time and temperature, and the amount of digestion enzyme per total protein ${ }^{24,49,50}$ ) will affect the protein quantification. The results in this study emphasize that all potential sources of variability should be considered and that more streamlined, generic methods for the quantification of membrane proteins needs to be established.

In conclusion, we found a large variability in concentrations of drug transporters and drug metabolizing enzymes obtained by different methods, with substantially lower levels found after subcellular fractionation compared to levels measured in whole tissue digests. In general, proteins were quantified to similar mean levels by methods using whole tissue digests. Therefore, when possible, whole cell or tissue preparations are recommended. However, a drawback with using whole tissue digests is matrix complexity, which may give lower resolution for low abundance proteins. Our results show that immunoprecipitation from whole tissue digests may improve the precision to that obtained in the less complex membrane 
fractions. We hope that our results will motivate further evaluation and standardization of proteomics methods for quantification of proteins important for drug disposition, and eventually contribute to improved pharmacokinetic modeling.

\section{ASSOCIATED CONTENT}

\section{S Supporting Information}

The Supporting Information is available free of charge on the ACS Publications website at DOI: 10.1021/acs.molpharmaceut.7b00364.

Detailed description of the proteomic methods, supporting figures and tables (PDF)

Protein concentrations of all quantified proteins in the ten liver samples obtained in the different laboratories (XLSX)

Criteria for MRM-quantification in the respective laboratory (XLSX)

\section{AUTHOR INFORMATION}

\section{Corresponding Author}

*E-mail: per.artursson@farmaci.uu.se; Phone: +46-18 47144 71.

\section{ORCID}

Christine Wegler: 0000-0002-2810-7518

Jacek R. Wiśniewski: 0000-0002-8452-5095

Brahim Achour: 0000-0002-2595-5626

\section{Author Contributions}

P.A. initiated the study. C.W., P.A, F.Z.G., T.A.B., J.R.W., D.B., S.O., O.P., T.O.J., B.A., A.R-H., E.vdS., and H.M.W. contributed to the study design. C.W., F.Z.G., J.R.W., C.G., D.B., S.O., A.N., F.W., H.S.H., O.P., B.A., E.vdS., and H.M.W. performed experiments and analyzed the results. C.W. compiled data and performed final data analyses. The manuscript was written by C.W. and P.A, and revised by C.W., P.A, F.Z.G., T.A.B., J.R.W., D.B., S.O., O.P., B.A., A.R-H., E.vdS., and H.M.W.

\section{Notes}

The authors declare no competing financial interest.

\section{ACKNOWLEDGMENTS}

C.W, F.Z.G., and P.A. are thankful to Elin Khan and Patrik Lundquist for lab support, and Aljona Saleh and Richard Svensson for MS support. The authors are grateful to Matthew Harwood for constructive discussion and to Katharina Zettl for brilliant technical assistance. P.A. and C.W. were supported by the Swedish Research Council (approval number 2822 and 5715). F.Z.G. was supported by the Swiss National Science Foundation grants P200P3_154635 and P2EZP3_148644. J.R.W. was supported by the German Research Foundation (DFG/Gottfried Wilhelm Leibniz Prize). T.O.J., O.P., F.W., and H.S.H. were funded by the German Federal Ministry of Education and Research (BMBF; grant FKZ 0315742,Virtual Liver) and grant FKZ 031A142 (GO-Bio). The work of S.O., D.B. and C.G. were funded by the German Federal Ministry of Education and Research (BMBF; grant 03IPT612X, InnoProfile-Transfer). A.R-H. and B.A. were supported by the Division of Pharmacy and Optometry, School of Health Sciences, University of Manchester, and acknowledge the Bio-MS Facility, University of Manchester, for instrument access and technical support with sample preparation.

\section{REFERENCES}

(1) Aebersold, R; Mann, M. Mass-spectrometric exploration of proteome structure and function. Nature 2016, 537 (7620), 347-55.

(2) Humphrey, S. J.; Azimifar, S. B.; Mann, M. High-throughput phosphoproteomics reveals in vivo insulin signaling dynamics. Nat. Biotechnol. 2015, 33 (9), 990-5.

(3) Deshmukh, A. S.; Murgia, M.; Nagaraj, N.; Treebak, J. T.; Cox, J.; Mann, M. Deep proteomics of mouse skeletal muscle enables quantitation of protein isoforms, metabolic pathways, and transcription factors. Mol. Cell. Proteomics 2015, 14 (4), 841-53.

(4) Williams, E. G.; Wu, Y.; Jha, P.; Dubuis, S.; Blattmann, P.; Argmann, C. A.; Houten, S. M.; Amariuta, T.; Wolski, W.; Zamboni, N.; Aebersold, R.; Auwerx, J. Systems proteomics of liver mitochondria function. Science 2016, 352 (6291), aad0189.

(5) Gillet, L. C.; Leitner, A.; Aebersold, R. Mass Spectrometry Applied to Bottom-Up Proteomics: Entering the High-Throughput Era for Hypothesis Testing. Annu. Rev. Anal. Chem. 2016, 9 (1), 44972.

(6) Wisniewski, J. R.; Zougman, A.; Nagaraj, N.; Mann, M. Universal sample preparation method for proteome analysis. Nat. Methods 2009, 6 (5), 359-62.

(7) Wisniewski, J. R.; Vildhede, A.; Noren, A.; Artursson, P. In-depth quantitative analysis and comparison of the human hepatocyte and hepatoma cell line HepG2 proteomes. J. Proteomics 2016, 136, 23447.

(8) Hughes, C. S.; Foehr, S.; Garfield, D. A.; Furlong, E. E.; Steinmetz, L. M.; Krijgsveld, J. Ultrasensitive proteome analysis using paramagnetic bead technology. Mol. Syst. Biol. 2014, 10, 757.

(9) Weiss, F.; Schnabel, A.; Planatscher, H.; van den Berg, B. H.; Serschnitzki, B.; Nuessler, A. K.; Thasler, W. E.; Weiss, T. S.; Reuss, M.; Stoll, D.; Templin, M. F.; Joos, T. O.; Marcus, K.; Poetz, O. Indirect protein quantification of drug-transforming enzymes using peptide group-specific immunoaffinity enrichment and mass spectrometry. Sci. Rep. 2015, 5, 8759.

(10) Kamiie, J.; Ohtsuki, S.; Iwase, R.; Ohmine, K.; Katsukura, Y.; Yanai, K.; Sekine, Y.; Uchida, Y.; Ito, S.; Terasaki, T. Quantitative atlas of membrane transporter proteins: development and application of a highly sensitive simultaneous LC/MS/MS method combined with novel in-silico peptide selection criteria. Pharm. Res. 2008, 25 (6), 1469-83.

(11) Li, N.; Bi, Y. A.; Duignan, D. B.; Lai, Y. Quantitative expression profile of hepatobiliary transporters in sandwich cultured rat and human hepatocytes. Mol. Pharmaceutics 2009, 6 (4), 1180-9.

(12) Miliotis, T.; Ali, L.; Palm, J. E.; Lundqvist, A. J.; Ahnoff, M.; Andersson, T. B.; Hilgendorf, C. Development of a highly sensitive method using liquid chromatography-multiple reaction monitoring to quantify membrane P-glycoprotein in biological matrices and relationship to transport function. Drug metabolism and disposition: the biological fate of chemicals 2011, 39 (12), 2440-9.

(13) Huber, L. A.; Pfaller, K.; Vietor, I. Organelle proteomics: implications for subcellular fractionation in proteomics. Circ. Res. 2003, 92 (9), 962-968.

(14) Groer, C.; Busch, D.; Patrzyk, M.; Beyer, K.; Busemann, A.; Heidecke, C. D.; Drozdzik, M.; Siegmund, W.; Oswald, S. Absolute protein quantification of clinically relevant cytochrome $\mathrm{P} 450$ enzymes and UDP-glucuronosyltransferases by mass spectrometry-based targeted proteomics. J. Pharm. Biomed. Anal. 2014, 100, 393-401.

(15) Kumar, V.; Prasad, B.; Patilea, G.; Gupta, A.; Salphati, L.; Evers, R.; Hop, C. E.; Unadkat, J. D. Quantitative transporter proteomics by liquid chromatography with tandem mass spectrometry: addressing methodologic issues of plasma membrane isolation and expressionactivity relationship. Drug Metab. Dispos. 2015, 43 (2), 284-8.

(16) van de Steeg, E.; Greupink, R.; Schreurs, M.; Nooijen, I. H.; Verhoeckx, K. C.; Hanemaaijer, R.; Ripken, D.; Monshouwer, M.; Vlaming, M. L.; DeGroot, J.; Verwei, M.; Russel, F. G.; Huisman, M. T.; Wortelboer, H. M. Drug-drug interactions between rosuvastatin and oral antidiabetic drugs occurring at the level of OATP1B1. Drug Metab. Dispos. 2013, 41 (3), 592-601. 
(17) Lesur, A.; Domon, B. Advances in high-resolution accurate mass spectrometry application to targeted proteomics. Proteomics 2015, 15 (5-6), 880-90.

(18) Picard, G.; Lebert, D.; Louwagie, M.; Adrait, A.; Huillet, C.; Vandenesch, F.; Bruley, C.; Garin, J.; Jaquinod, M.; Brun, V. PSAQ standards for accurate MS-based quantification of proteins: from the concept to biomedical applications. J. Mass Spectrom. 2012, 47 (10), 1353-63.

(19) Domon, B.; Aebersold, R. Options and considerations when selecting a quantitative proteomics strategy. Nat. Biotechnol. 2010, 28 (7), 710-21.

(20) Campos, J. M.; Neves, L. X.; de Paiva, N. C.; de Oliveira e Castro, R. A.; Case, A. H.; Carneiro, C. M.; Andrade, M. H.; CastroBorges, $\mathrm{W}$. Understanding global changes of the liver proteome during murine schistosomiasis using a label-free shotgun approach. J. Proteomics 2017, 151, 193-203.

(21) Wisniewski, J. R; Rakus, D. Multi-enzyme digestion FASP and the 'Total Protein Approach'-based absolute quantification of the Escherichia coli proteome. J. Proteomics 2014, 109, 322-31.

(22) Harwood, M. D.; Russell, M. R.; Neuhoff, S.; Warhurst, G.; Rostami-Hodjegan, A. Lost in centrifugation: accounting for transporter protein losses in quantitative targeted absolute proteomics. Drug Metab. Dispos. 2014, 42 (10), 1766-72.

(23) Piehowski, P. D.; Petyuk, V. A.; Orton, D. J.; Xie, F.; Moore, R. J.; Ramirez-Restrepo, M.; Engel, A.; Lieberman, A. P.; Albin, R. L.; Camp, D. G.; Smith, R. D.; Myers, A. J. Sources of technical variability in quantitative LC-MS proteomics: human brain tissue sample analysis. J. Proteome Res. 2013, 12 (5), 2128-37.

(24) Prasad, B.; Unadkat, J. D. Optimized approaches for quantification of drug transporters in tissues and cells by MRM proteomics. AAPS J. 2014, 16 (4), 634-48.

(25) Scott, K. B.; Turko, I. V.; Phinney, K. W. Quantitative performance of internal standard platforms for absolute protein quantification using multiple reaction monitoring-mass spectrometry. Anal. Chem. 2015, 87 (8), 4429-35.

(26) Wang, D.; Guo, Y.; Wrighton, S. A.; Cooke, G. E.; Sadee, W. Intronic polymorphism in CYP3A4 affects hepatic expression and response to statin drugs. Pharmacogenomics J. 2011, 11 (4), 274-86.

(27) Achour, B.; Barber, J.; Rostami-Hodjegan, A. Expression of hepatic drug-metabolizing cytochrome p450 enzymes and their intercorrelations: a meta-analysis. Drug Metab. Dispos. 2014, 42 (8), 1349-56.

(28) Achour, B.; Rostami-Hodjegan, A.; Barber, J. Protein expression of various hepatic uridine $5^{\prime}$-diphosphate glucuronosyltransferase (UGT) enzymes and their inter-correlations: a meta-analysis. Biopharm. Drug Dispos. 2014, 35 (6), 353-61.

(29) Vildhede, A.; Wisniewski, J. R.; Noren, A.; Karlgren, M.; Artursson, P. Comparative Proteomic Analysis of Human Liver Tissue and Isolated Hepatocytes with a Focus on Proteins Determining Drug Exposure. J. Proteome Res. 2015, 14 (8), 3305-14.

(30) Tang, H.; Hussain, A.; Leal, M.; Mayersohn, M.; Fluhler, E. Interspecies prediction of human drug clearance based on scaling data from one or two animal species. Drug metabolism and disposition: the biological fate of chemicals 2007, 35 (10), 1886-93.

(31) Karlgren, M.; Vildhede, A.; Norinder, U.; Wisniewski, J. R.; Kimoto, E.; Lai, Y.; Haglund, U.; Artursson, P. Classification of inhibitors of hepatic organic anion transporting polypeptides (OATPs): influence of protein expression on drug-drug interactions. J. Med. Chem. 2012, 55 (10), 4740-63.

(32) Dobson, L.; Remenyi, I.; Tusnady, G. E. The human transmembrane proteome. Biol. Direct 2015, 10, 31.

(33) Fagerberg, L.; Jonasson, K.; von Heijne, G.; Uhlen, M.; Berglund, L. Prediction of the human membrane proteome. Proteomics 2010, 10 (6), 1141-9.

(34) Wisniewski, J. R.; Wegler, C.; Artursson, P. Subcellular fractionation of human liver reveals limits in global proteomic quantification from isolated fractions. Anal. Biochem. 2016, 509, 82-8.
(35) Itzhak, D. N.; Tyanova, S.; Cox, J.; Borner, G. H. Global, quantitative and dynamic mapping of protein subcellular localization. eLife 2016, 5, e16950.

(36) Wisniewski, J. R.; Dus, K.; Mann, M. Proteomic workflow for analysis of archival formalin-fixed and paraffin-embedded clinical samples to a depth of 10000 proteins. Proteomics: Clin. Appl. 2013, 7 (3-4), 225-33.

(37) Zanger, U. M.; Fischer, J.; Raimundo, S.; Stuven, T.; Evert, B. O.; Schwab, M.; Eichelbaum, M. Comprehensive analysis of the genetic factors determining expression and function of hepatic CYP2D6. Pharmacogenetics 2001, 11 (7), 573-85.

(38) Suski, J. M.; Lebiedzinska, M.; Wojtala, A.; Duszynski, J.; Giorgi, C.; Pinton, P.; Wieckowski, M. R. Isolation of plasma membraneassociated membranes from rat liver. Nat. Protoc. 2014, 9 (2), 312-22.

(39) Mukhopadhyay, A.; Nieves, E.; Che, F. Y.; Wang, J.; Jin, L.; Murray, J. W.; Gordon, K.; Angeletti, R. H.; Wolkoff, A. W. Proteomic analysis of endocytic vesicles: Rabla regulates motility of early endocytic vesicles. J. Cell Sci. 2011, 124 (5), 765-75.

(40) Christoforou, A.; Mulvey, C. M.; Breckels, L. M.; Geladaki, A.; Hurrell, T.; Hayward, P. C.; Naake, T.; Gatto, L.; Viner, R.; Martinez Arias, A.; Lilley, K. S. A draft map of the mouse pluripotent stem cell spatial proteome. Nat. Commun. 2016, 7, 9992.

(41) Ohtsuki, S.; Ikeda, C.; Uchida, Y.; Sakamoto, Y.; Miller, F.; Glacial, F.; Decleves, X.; Scherrmann, J. M.; Couraud, P. O.; Kubo, Y.; Tachikawa, M.; Terasaki, T. Quantitative targeted absolute proteomic analysis of transporters, receptors and junction proteins for validation of human cerebral microvascular endothelial cell line hCMEC/D3 as a human blood-brain barrier model. Mol. Pharmaceutics 2013, 10 (1), 289-96.

(42) IJsselstijn, L.; Stoop, M. P.; Stingl, C.; Sillevis Smitt, P. A.; Luider, T. M.; Dekker, L. J. Comparative study of targeted and labelfree mass spectrometry methods for protein quantification. J. Proteome Res. 2013, 12 (4), 2005-11.

(43) Mei, H.; Hsieh, Y.; Nardo, C.; Xu, X.; Wang, S.; Ng, K.; Korfmacher, W. A. Investigation of matrix effects in bioanalytical highperformance liquid chromatography/tandem mass spectrometric assays: application to drug discovery. Rapid Commun. Mass Spectrom. 2003, 17 (1), 97-103.

(44) Rowland, M.; Peck, C.; Tucker, G. Physiologically-based pharmacokinetics in drug development and regulatory science. Annu. Rev. Pharmacol. Toxicol. 2011, 51, 45-73.

(45) Zhuang, X.; Lu, C. PBPK modeling and simulation in drug research and development. Acta Pharm. Sin. B 2016, 6 (5), 430-440.

(46) Lund-Johansen, F.; de la Rosa Carrillo, D.; Mehta, A.; Sikorski, K.; Inngjerdingen, M.; Kalina, T.; Roysland, K.; de Souza, G. A.; Bradbury, A. R.; Lecrevisse, Q.; Stuchly, J. MetaMass, a tool for metaanalysis of subcellular proteomics data. Nat. Methods 2016, 13 (10), 837-40.

(47) Kwiatkowski, M.; Wurlitzer, M.; Krutilin, A.; Kiani, P.; Nimer, R.; Omidi, M.; Mannaa, A.; Bussmann, T.; Bartkowiak, K.; Kruber, S.; Uschold, S.; Steffen, P.; Lubberstedt, J.; Kupker, N.; Petersen, H.; Knecht, R.; Hansen, N. O.; Zarrine-Afsar, A.; Robertson, W. D.; Miller, R. J.; Schluter, H. Homogenization of tissues via picosecond-infrared laser (PIRL) ablation: Giving a closer view on the in-vivo composition of protein species as compared to mechanical homogenization. $J$. Proteomics 2016, 134, 193-202.

(48) Butt, R. H.; Coorssen, J. R. Pre-extraction sample handling by automated frozen disruption significantly improves subsequent proteomic analyses. J. Proteome Res. 2006, 5 (2), 437-48.

(49) Moore, S. M.; Hess, S. M.; Jorgenson, J. W. Extraction, Enrichment, Solubilization, and Digestion Techniques for Membrane Proteomics. J. Proteome Res. 2016, 15 (4), 1243-52.

(50) Wisniewski, J. R; Mann, M. Consecutive proteolytic digestion in an enzyme reactor increases depth of proteomic and phosphoproteomic analysis. Anal. Chem. 2012, 84 (6), 2631-7. 